\title{
Effects of Some Respiratory and Glycolytic Inhibitors on Mitochondrial Functionality in Bovine Semen
}

\author{
S.K. Shahani ${ }^{1}$, S.G. Revell ${ }^{2}$, C.McG. Argo ${ }^{3}$ and R.D. Murray*,1 \\ ${ }^{I}$ University of Liverpool Institute of Translational Medicine, Leahurst Campus, Chester High Road, Neston. CH64 7TE, \\ UK; ${ }^{2}$ Genus Breeding Limited, Freezing Unit, Llanrhydd, Ruthin, Denbighshire LL15 2UP, UK; ${ }^{3}$ University of \\ Liverpool Institute of Ageing and Chronic Disease, Leahurst Campus, Chester High Road, Neston. CH64 7TE, UK.
}

\begin{abstract}
Fertility potential of spermatozoa depends on maintenance of the mitochondrial membrane potential $(\Delta \Psi \mathrm{m})$ that provides energy for sperm hyperactivation immediately prior to successful fertilization. Mitochondrial structure and integrity are associated with sperm motility and reduced fertility, and measurement of $\Delta \Psi \mathrm{m}$ may be suitable for determining bull semen quality. Mitochondrial membrane potential in four commercial AI bulls was assessed using JC-1 and propidium iodide in the presence of the glycolytic inhibitors 2-deoxy-D-glucose (DOG) and iodoacetamide (IAM) and the respiratory inhibitor valinomycin (VAL) to determine the maximum $\Delta \Psi \mathrm{m}$ at minimum incubation. Flow cytometry recorded $\Delta \Psi \mathrm{m}$ within a unified population for all treatments that represented sperm with low and high $\Delta \Psi \mathrm{m}$ respectively. Maximum $\Delta \Psi \mathrm{m}$ was seen at $40 \mathrm{~min}$ incubation. Mean high fluorescence intensity (MFI) (orange) was significantly greater for untreated compared to the treated sperm, at 40 and 80 min incubation, in both fresh and frozenthawed semen. In sperm treated with VAL and IAM, $\triangle \Psi \mathrm{m}$ was lowered significantly, and the proportion of sperm with high: low $\triangle \Psi \mathrm{m}$ ratio was higher in control and DOG- treated samples representing more active mitochondria. In samples treated with VAL and IAM, the ratio was reversed, representing loss in activity. Cryopreservation increased the high:low $\Delta \Psi \mathrm{m}$ ratio only in bull 1 by $30 \%$ and lowered it in bulls 2,3 and 4 by $20 \%-70 \%$ compared to fresh semen. The rise in bull 1 may relate to be the product of sperm demonstrating a capacitation- like effect of the freeze-thaw process which stimulated sperm hyperactivity.
\end{abstract}

We conclude that mitochondrial function was affected adversely on freeze-thaw process. The 40 min incubation is satisfactory for future studies. Furthermore, IAM, a known glycolytic inhibitor, has a similar effect to VAL, a recognized respiratory inhibitor, which should provide a basis for further research.

Keywords: Mitochondria, cryo-preservation, bull semen, flow cytometry.

\section{INTRODUCTION}

Cryopreservation alters the integrity and functionality of some sperm through damage to the plasma and acrosomal membranes and associated disruption of mitochondrial function $[1,2]$. Consequently, the percentage of fully functional sperm with intact cell and organelle membranes is reduced after the freeze-thawing process [3]. On freezing, ice crystals form in the extra-cellular medium, increasing the osmolarity of the unfrozen solution. As intra-cellular water diffuses out in response to this change in osmotic gradient, the cell and plasma membranes become dehydrated [4]. At thawing, this phenomenon is reversed as the extra-cellular ice crystals melt and water re-enters the sperm. The ionic permeability and enzyme activity within the plasma membrane are disrupted creating lipid phase transitions at around $17-36^{\circ} \mathrm{C}$ [5-7]. Working with boar semen Guthrie et al., [8] increased the extender osmolarity from 300 to $600 \mathrm{mOsm} / \mathrm{kg}$, and found a $50 \%$ reduction in mitochondrial membrane potential. After freeze/thaw, Bilodeau et al., [9] found that sperm glutathione (GSH) concentration was

*Address correspondence to this author at the University of Liverpool Institute of Translational Medicine, Leahurst Campus, Chester High Road, Neston. CH64 7TE, UK; Tel.: +44-151- 794- 6056;

E-mail: richmu@liverpool.ac.uk reduced by $78 \%$ and superoxide dismutase activity by $50 \%$, suggesting that oxidative stress had occurred. Premature ageing and capacitation have been associated with impaired acrosomal and/or mitochondrial membrane integrity, lowering ATP synthesis and reducing motility [10], as does bacterial contamination within an ejaculate [11].

Mitochondrial respiratory activity has been investigated in vitro using drugs that uncouple oxidative phosphorylation (OXPHOS), either by inhibiting electron transport chain (ETC) or blocking the essential link between the respiratory pathways and phosphorylation. One such drug, carbonyl cyanide m-chlorophenylhydrazone, markedly reduced mitochondrial membrane potential $(\Delta \Psi \mathrm{m})$ in both mouse and boar spermatozoa [8, 12]. A similar reduction has been observed in human sperm, treated with potassium cyanide that blocks cytochrome $\mathrm{C}$ oxidase within complex IV of the ETC, or p-trifluoromethoxy carbonyl cyanide phenylhydrazone, and valinomycin that both uncouple OXPHOS [13].

ATP synthesis pathways may be investigated in sperm using a number of specific enzyme inhibitors. One example is 2-deoxy-D-glucose (DOG), a competitive inhibitor of glycolysis that targets hexokinase within the reaction glucose glucose 6-phosphate by competing with glucose during phosphorylation: the resulting product is DOG-6-phosphate 
which cannot be metabolized further $[14,15]$. When bovine sperm are re-suspended in a medium containing DOG and pyruvate and incubated at $37^{\circ} \mathrm{C}$ for 10 minutes, at least $70 \%$ of sperm cells remain motile, demonstrating that spermatozoa maintain motility utilizing mitochondrial ATP without employing the glycolytic pathway at this level [16] . By comparison, mouse sperm incubated similarly show a significant reduction in their motility, even in the presence of pyruvate and lactate $[12,15]$ : this suggests that glycolytic ATP is crucial for sperm motility, or the decrease in motility might be due to utilization of mitochondrial ATP by DOG when phosphorylated with hexokinase. Investigating mitochondrial membrane potential in mouse sperm labelled with JC-1, Mukai and Okuno [12] found no change in $\Delta \Psi \mathrm{m}$ in the presence of DOG and either glucose or pyruvate substrates, suggesting that DOG had no effect on mitochondrial respiration.

Another glycolytic inhibitor is iodoacetamide (IAM) which inactivates the enzyme glyceraldehyde-3-phosphate dehydrogenase (GAPDH) by binding irreversibly to cysteine residues [15]. IAM can deprive rat astrocytes of glutathione (GSH), inhibit cellular GAPDH activity, and reduce cell lactate synthesis. It may cause cell death after a long incubation period [17] and should not be used to inactivate GAPDH and interrupt glycolysis because it lowers the GSH content of cultured cells. IAM has no effect on sperm ATP concentrations and motility if oxidizable substrate is provided but it does lower ATP concentrations and motility in the presence of glucose suggested that IAM had no effect on mitochondrial ATP that sperm utilize to maintain their motility, but it interrupted glycolysis, preventing an adequate supply of respiratory substrate for mitochondria, thereby decreasing ATP synthesis and motility [15].

Another group of drugs that can be used to investigate mitochondrial function are the $\mathrm{K}^{+}$ionophores. One of these is valinomycin (VAL), a dodecadepsipeptide that induces mitochondrial swelling especially in the presence of acetate [18] through an increased uptake of $\mathrm{K}^{+}$. Inhibition of ETC in mitochondria occurs, due to loss of inner membrane potential, oxidation of pyridine nucleotides, and induction of apoptosis [19]. In nature, dodecadepsipeptides are found as a cellular toxins produced by several Streptomyces species of bacteria and have a similar effect on mitochondrial activity as VAL [20]. VAL is a passive carrier for $\mathrm{K}^{+}$, when it encounters the cell membrane surface: it transports these ions across membranes, thereby inhibiting oxidative phosphorylation by antagonizing the proton motive force. To give a true measure of mitochondrial activity sperm incubated with VAL should produce a base fluorescence value, which can be subtracted from, or compared with, that for the same sample incubated without VAL.

In our preliminary experiments to obtain maximum uptake of JC-1 in sperm population at minimum incubation time, variable results were observed with same bull examined on different days as well as fresh and post thaw semen. Selection of motile sperm by Bovipure showed maximum $\Delta \Psi \mathrm{m}$ at 30 minutes in fresh and post-thaw sperm labelled with JC-1, while maximum $\Delta \Psi \mathrm{m}$ was observed at 150 and $90 \mathrm{~min}$ corresponding fresh and post-thaw semen washed with Ruthin extender, a glycolysis supporting medium (data not shown). Uprating of $\Delta \Psi \mathrm{m}$ in motile sperm of $3 / 4$ bulls was seen in post-thaw semen. This was consistent with the results of Garrett et al., [21] who observed higher oxygen consumption rate in post-thaw semen. Whereas, semen washed in Ruthin medium: $\Delta \Psi \mathrm{m}$ has been increased with increase in incubation time in all bulls of fresh sperm and $2 / 6$ bulls of post-thaw sperm respectively. Uprating of $\Delta \Psi \mathrm{m}$ in bulls might be sperm that initially were dependent on ATP generated by glycolysis accumulating its end product pyruvate/lactic acid, later with increase in incubation time the sperm demonstrated capacitation like changes and became hyperactive [22], which result in increased demand for ATP and relied on mitochondrial ATP for long time.

This paper investigated the effect of freeze/thawing on sperm $\Delta \Psi \mathrm{m}$ obtained from bulls incubated in the presence of respiratory and glycolysis inhibitors. The use of these inhibitors may help to determine the maximum mitochondrial potential at a minimum incubation time after restricting glycolysis activity, thus forcing the sperm to derive their energy from respiration and also the extent of background fluorescence by comparing fluorescence when incubated with and without inhibitors.

\section{MATERIALS AND METHODS}

Four treatments were examined using JC-1 and propidium iodide (PI): control and $90 \mathrm{nM}$ valinomycin (VAL) providing $44 \mathrm{mM}$ sorbitol as a glycolytic substrate and $5 \mathrm{mM}$ 2-deoxy-D-glucose (DOG) and $0.5 \mathrm{mM}$ iodoacetamide (IAM) providing $9 \mathrm{mM}$ pyruvic acid as a respiratory substrate.

\section{Preparation of Fresh and Frozen/Thawed Semen}

Ejaculates were collected from four mature commercial AI bulls using an AV. From each bull, an aliquot of 1-2 ml of raw semen after first dilution in $20 \%$ egg yolk-trisglycerol (EYTG) extender was taken according to semen concentration range $400-800 \times 10^{6} / \mathrm{ml}$ in a centrifuge tube. Phosphate-buffered saline (PBS) was added to give a volume of $10 \mathrm{ml}$. This suspension was centrifuged at $600 \mathrm{x} g$ for 10 minutes at room temperature. The supernatant was removed and the resulting pellet transferred to a fresh test tube in a water bath at $39^{\circ} \mathrm{C}$. PBS extender was added to the pellet to give a suspension volume of $2 \mathrm{ml}$ and the resulting concentration of sperm was estimated using a nucleocounter (Chemometec A/S, Denmark). The concentration was adjusted to $24 \times 10^{6}$ per $\mathrm{ml}$ by adding warmed Ruthin extender to Control and VAL and PBS extender to DOG and IAM treatments (Fig. 1). 'Ruthin' extender is the same as the 'Reading' extender [23] except for the substitution of sorbitol for glucose and the removal of trehalose [24]. From this suspension, $250 \mu 1$ from each in duplicate for each treatment was removed for investigation that contained $6 \times 10^{6}$ sperm. $250 \mu 1$ of Ruthin extender and DOG and IAM suspension were added to semen as per treatment. Samples were labelled as Control (C1, C2), Valinomycin (VAL1, VAL2), 2-deoxy-D-glucose (DOG1, DOG2) and Iodoacetamide (IAM1, IAM2). The labelled samples were left for 15 minutes before staining.

Thirty straws, each containing $0.25 \mathrm{ml}$, of frozen bull semen were received in liquid nitrogen and thawed by immersing them in a water bath at $39^{\circ} \mathrm{C}$ for 30 secs. The 
contents of each straw were transferred into a centrifuge tube in water bath at $39^{\circ} \mathrm{C}$ and thereafter processed as for the fresh semen (Fig. 1).

\section{Fluorescence Staining of Sperm and Flow Cytometry}

After semen preparation, $360 \mu \mathrm{l}$ was taken from $500 \mu \mathrm{l}$ semen into fresh test tube and $40 \mu \mathrm{l}$ of JC-1 were added, together with $4 \mu \mathrm{l}$ VAL as appropriate (Fig. 1), approximately 2 minutes apart to accommodate running time on the flow cytometer. Each sample was incubated at $39^{\circ} \mathrm{C}$ for 40 and $80 \mathrm{~min}$ and $40 \mu \mathrm{l}$ of JC-1 incubated stirred semen from each treatment was added to $995 \mu \mathrm{l}$ of warmed Ruthin/PBS extender as per treatment and $5 \mu \mathrm{PI}$ in flow tube (Fig. 1). The readings were then taken at each incubation time. The final stain concentrations of JC-I and PI were $0.1 \mu \mathrm{M}$ and $12 \mu \mathrm{M}$ respectively. Samples were analyzed on a Partec Cyflow Space Flow Cytometer (Partec GmbH, Gorlitz, Germany). Regional and logical gates were set to select live sperm population and exclude non-sperm specific events and dead cells.

\section{Statistical Analysis}

The real time data acquisition, analysis, and display was performed using FloMax ${ }^{\circledR}$, the PC based FCM software. Mean fluorescence intensity measured in Relative Fluorescence Units (RFU) of green (low $\Delta \Psi \mathrm{m}$ ) and orange/red (high $\Delta \Psi \mathrm{m}$ ) appeared in log-X and log-Y respectively, stored in an Excel spreadsheet. After cleaning the data, differences between fresh and frozen-thawed samples, within and between bulls after incubation with or without inhibitors were investigated using ANOVA, applying Tukey and Dunnett's comparison test within a general linear model procedure in Minitab16. Significant differences was accepted when $P<0.05$.

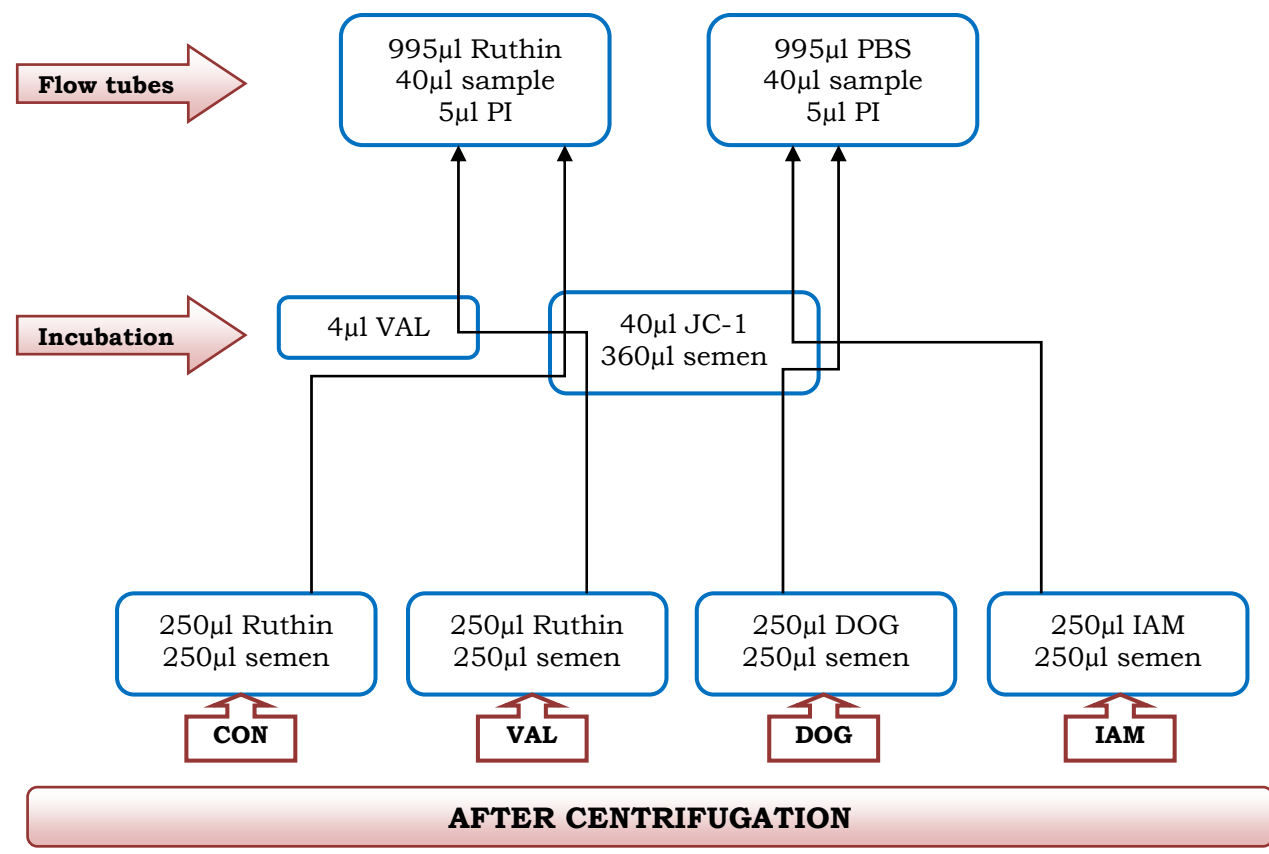

Fig. (1). Flow chart for JC-I and PI protocol after treatment with control (CON), valinomycin (VAL), 2-deoxy-D-glucose (DOG) and iodoacetamide (IAM).

\section{RESULTS}

Flow cytometry recording of mitochondrial membrane potential in fresh and frozen-thawed sperm incubated with JC-1, presented all dot plots within one single population in CON, VAL, DOG and IAM treatments. This one parameter fluorescence display included both green and orange fluorescence but to varying degrees representing sperm with low and high $\Delta \Psi \mathrm{m}$ respectively (Fig. 2). The orange and green populations in dot plots were difficult to separate. Therefore orange:green (high $\Delta \Psi \mathrm{m}$ : low $\Delta \Psi \mathrm{m}$ ) fluorescence ratio was performed to integrate green and orange fluorescence value in fresh and freeze-thaw semen.

\section{Comparison of Control (CON) with Treatment}

To compare $\Delta \Psi \mathrm{m}$ of untreated spermatozoa in a medium supporting glycolysis $(\mathrm{CON})$ with the treated spermatozoa after suspending their anaerobic (DOG, IAM) and aerobic (VAL) metabolic pathways: mean fluorescence intensity (MFI) of monomers was significantly higher in IAM and VAL and DOG and VAL as compare to CON at $40 \mathrm{~min}$ incubation in spermatozoa of fresh and freeze-thaw semen respectively (Figs. 3a \& 4a). Similar trend at 80 minutes incubation was seen in all treatments of fresh and freezethaw (except IAM) semen (Figs. 3b \& 4b). In contrast MFI of aggregates appeared to be significantly higher in $\mathrm{CON}$ as compared to IAM and VAL treatments at $40 \mathrm{~min}$ incubation in fresh (excluding VAL) and freeze-thaw semen and only against IAM at $80 \mathrm{~min}$ incubation in sperm of fresh and freeze-thaw semen (Figs. 3ab \& 4ab). A shift in $\Delta \Psi \mathrm{m}$ from high to low was found in samples treated with VAL and IAM. The high: low $\Delta \Psi \mathrm{m}(\mathrm{h} \Delta \Psi \mathrm{m}: 1 \Delta \Psi \mathrm{m})$ ratio was higher in $\mathrm{CON}$ and DOG treated samples representing more active mitochondria, while in samples treated with VAL and IAM the $\mathrm{h} \Delta \Psi \mathrm{m}: 1 \Delta \Psi \mathrm{m}$ fluorescence values were reversed to distinctly lower representing less active mitochondria at both incubation times in fresh and freeze-thaw semen (Figs. 3ab \& 4ab). High: low $\Delta \Psi \mathrm{m}$ ratio was significantly higher in

sample 


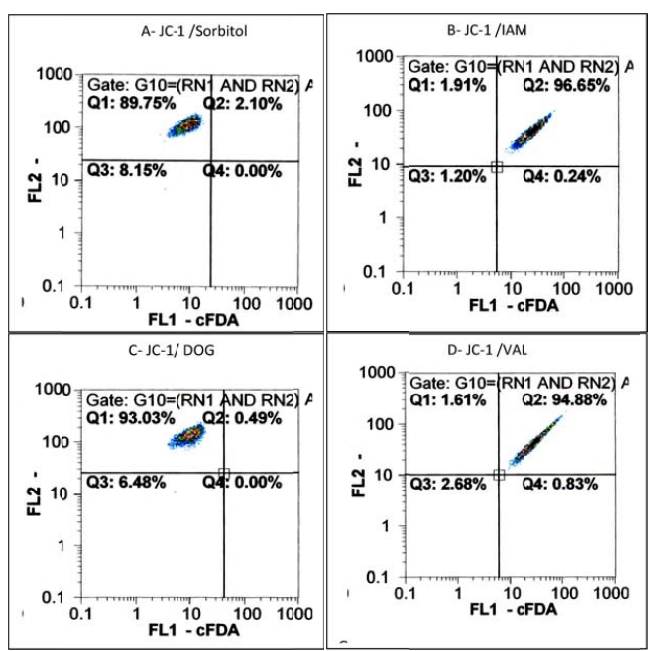

Fig. (2). Dot plots resulting from flow cytometric analyses of spermatozoa stained with JC-1. Dot plot of population with highly functional mitochondria displayed oval outline (A, C) and elongated in less functional mitochondria (B, D). A) population in presence of glycolytic substrate. B) population treated with IAM in presence of pyruvate. C) population treated with DOG in presence of pyruvate. D) population treated with VAL in presence of glycolytic substrate.
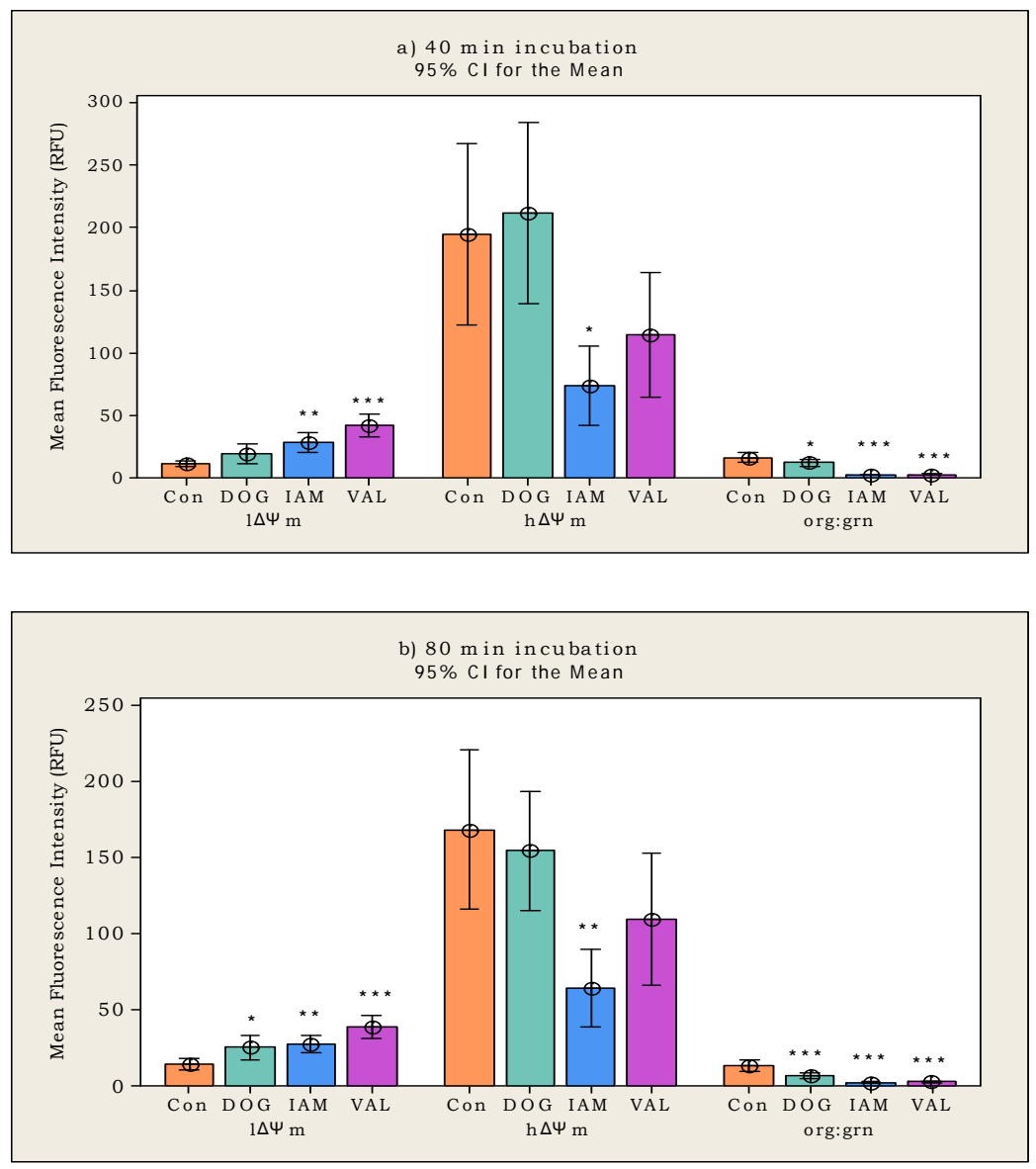

Fig. (3). (a \& b) Comparison of control with treatments of fresh for 40 and 80 minutes incubation times of low and high mitochondrial membrane potential $(1 \Delta \Psi \mathrm{m}$ and $\mathrm{h} \Delta \Psi \mathrm{m})$ and $\mathrm{h} \Delta \Psi \mathrm{m}: 1 \Delta \Psi \mathrm{m}$ ratio. Bars with the superscripts are significantly different to control; $*=\mathrm{p}<0.05$; $* *=\mathrm{p}<0.01$ and $* * *=\mathrm{p}<0.001$.

CON than other treatments in all incubation times and semen. To get a true measure of JC-1, after subtracting the $\mathrm{h} \Delta \Psi \mathrm{m}: 1 \Delta \Psi \mathrm{m}$ ratio of VAL from the $\mathrm{h} \Delta \Psi \mathrm{m}: 1 \Delta \Psi \mathrm{m}$ ratio of $\mathrm{CON}$ at both incubation times (data not presented), it was revealed that even though VAL causes a significant reduction in sperm mitochondrial activity, this has not been abolished completely. 

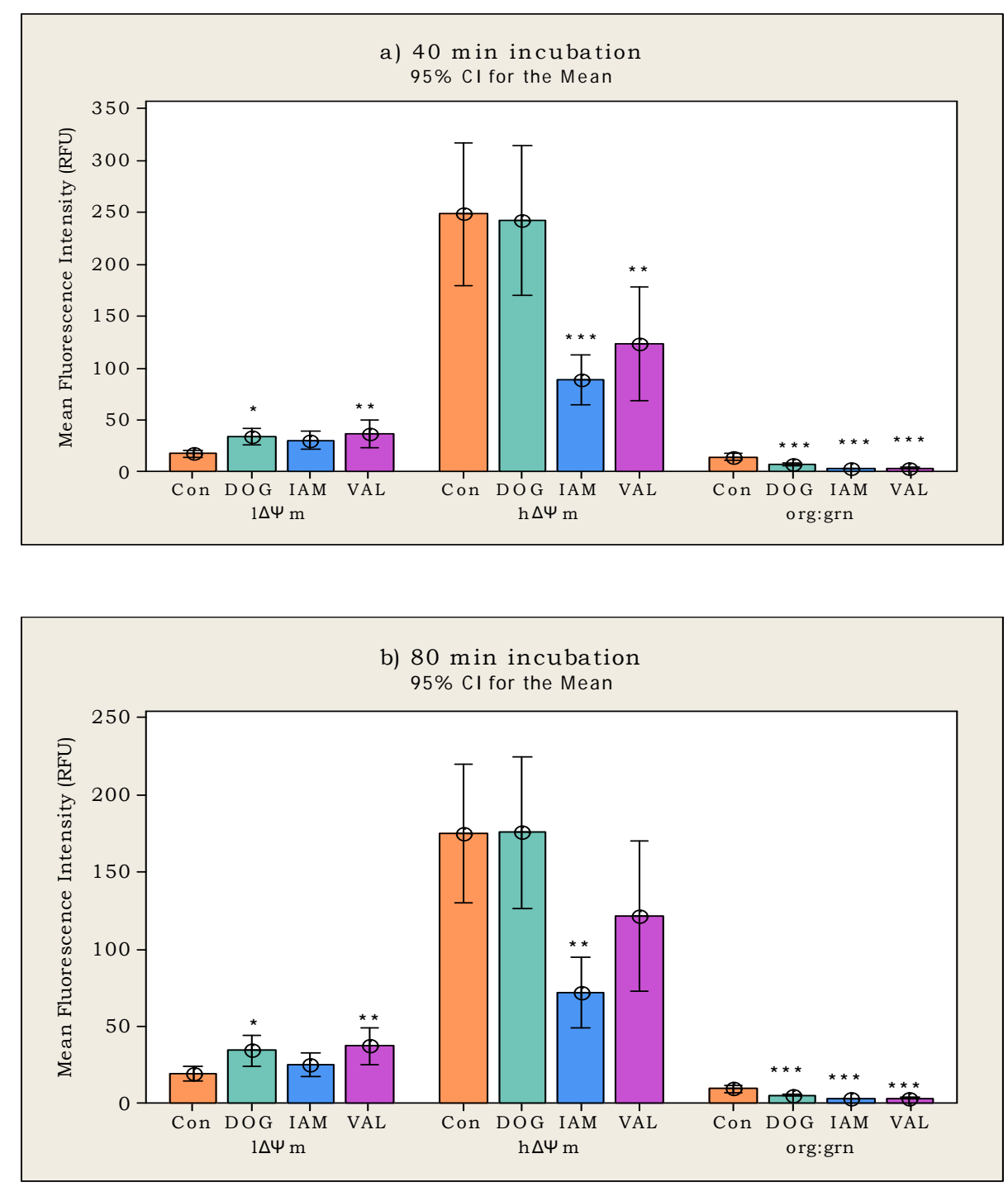

Fig. (4). (a \& b) Comparison of control with treatments of frozen-thawed semen for 40 and 80 minutes incubation times of low and high mitochondrial membrane potential $(1 \Delta \Psi \mathrm{m}$ and $\mathrm{h} \Delta \Psi \mathrm{m})$ and $\mathrm{h} \Delta \Psi \mathrm{m}: 1 \Delta \Psi \mathrm{m}$ ratio. Bars with the superscripts are significantly different to control; $*=\mathrm{p}<0.05 ; * *=\mathrm{p}<0.01$ and $* * *=\mathrm{p}<0.001$.

\section{Comparison Between Incubation Times Within Bull}

Although no significant differences were observed in $\mathrm{h} \Delta \Psi \mathrm{m}: 1 \Delta \Psi \mathrm{m}$ ratio between 40 and $80 \mathrm{~min}$ incubation times within each treatment of fresh and freeze-thaw semen except $\mathrm{h} \Delta \Psi \mathrm{m}: 1 \Delta \Psi \mathrm{m}$ ratio of Con freeze-thaw semen and DOG fresh and freeze-thaw semen (Fig. 5). However, maximum $\mathrm{h} \Delta \Psi \mathrm{m}: 1 \Delta \Psi \mathrm{m}$ ratio was seen at $40 \mathrm{~min}$ in all treatments and semen, but slightly lowered with VAL, fresh semen. When incubation time was compared within bulls, there was a reduction of $\mathrm{h} \Delta \Psi \mathrm{m}: \mathrm{I} \Delta \Psi \mathrm{m}$ ratio over time in untreated sperm (CON) of fresh and fresh-thaw semen of all bulls excluding bull 3 fresh semen (Fig. 6). These variations in $\mathrm{h} \Delta \Psi \mathrm{m}: 1 \Delta \Psi \mathrm{m}$ values were only significant within bull 2 fresh semen and bull 3 and bull 4 freeze-thaw semen.

\section{Comparison Between Fresh and Frozen-Thawed Semen Within Bulls}

In this section the results are presented at $40 \mathrm{~min}$ incubation because the maximum $\mathrm{h} \Delta \Psi \mathrm{m}: 1 \Delta \Psi \mathrm{m}$ ratio was observed at this point. The effect of cryopreservation on the proportion of $\mathrm{h} \Delta \Psi \mathrm{m}: 1 \Delta \Psi \mathrm{m}$ in identical samples of sperm before and after cryopreservation in the presence of glycolysis supported medium (CON), glycolysis (DOG, IAM) and respiratory (VAL) inhibitors stained with JC-1 observed variable results (Fig. 7a \& b). Cryopreservation increased $\mathrm{h} \Delta \Psi \mathrm{m}: 1 \Delta \Psi \mathrm{m}$ ratio only in bull $1(30 \%)$ and decreased in bulls 2 (70\%), 3 (20\%) and 4 (30\%) compared to fresh semen. The rise in bull 1 may be the product of sperm demonstrated capacitation like changes and became hyperactive [22], which result in increase in demand for ATP and relied on mitochondrial ATP for long time. Whereas decrease in $\mathrm{h} \Delta \Psi \mathrm{m}: 1 \Delta \Psi \mathrm{m}$ ratio in remaining bulls may be the involvement of an oxidative stress due to significant drop in antioxidant level during a freeze/thaw cycle [9]. Freeze-thaw also caused drop in $\mathrm{h} \Delta \Psi \mathrm{m}: 1 \Delta \Psi \mathrm{m}$ ratio in all bulls' sperm in presence of DOG that forced sperm to generate their energy exclusively via mitochondria (Fig. 7a \& b). In contrast $\mathrm{h} \Delta \Psi \mathrm{m}: 1 \Delta \Psi \mathrm{m}$ ratio of all bulls seemed to be higher in freezethaw sperm treated with VAL but comparable in bull 4 (Fig. 7a \& b). Interestingly, similar change in $\mathrm{h} \Delta \Psi \mathrm{m}: 1 \Delta \Psi \mathrm{m}$ close to VAL observed with glycolysis intermediate inhibitor IAM (Fig. 7a \& b). This variation in $\mathrm{h} \Delta \Psi \mathrm{m}: 1 \Delta \Psi \mathrm{m}$ ratio likely to 


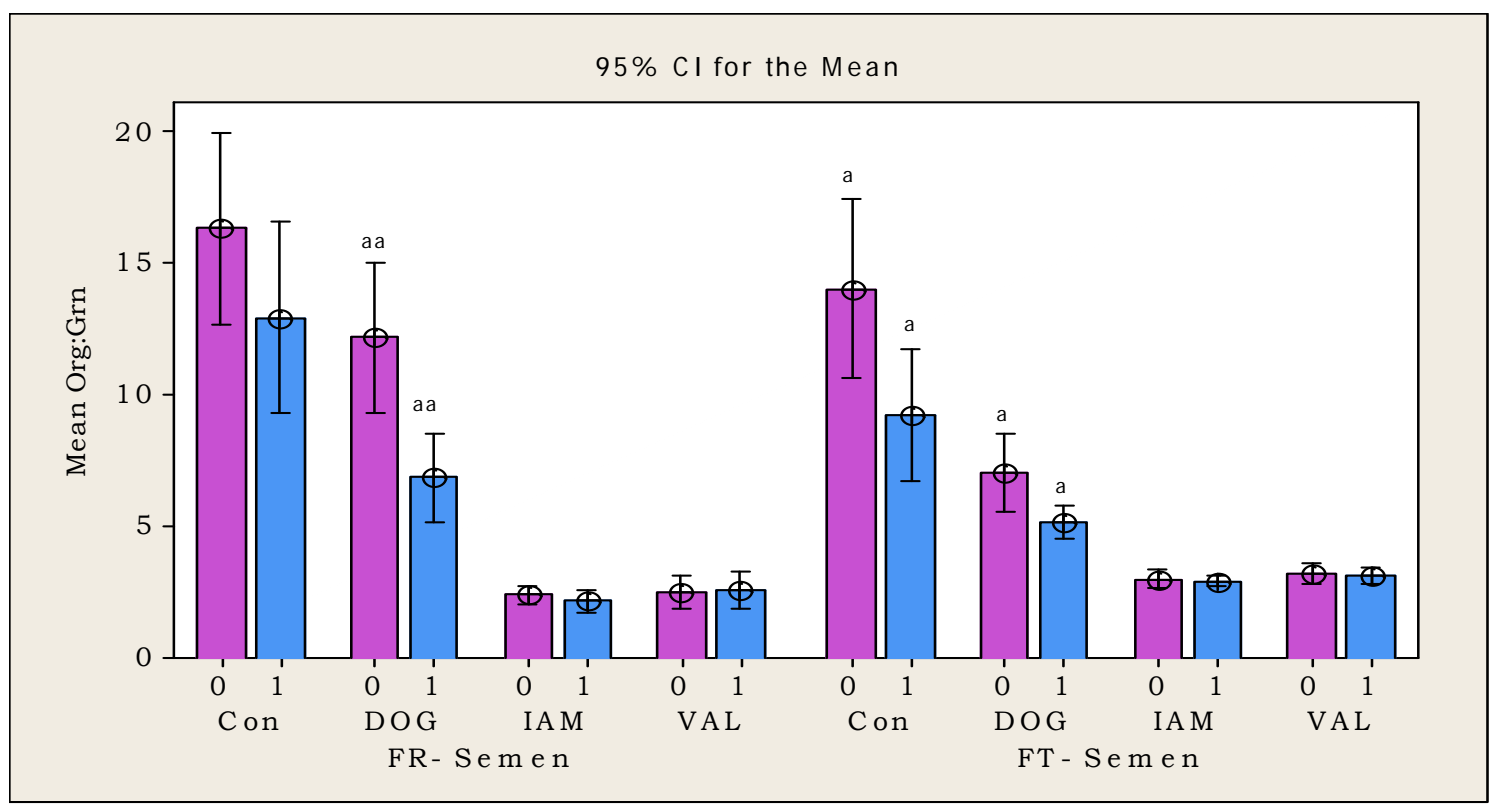

Fig. (5). Comparison of high: low $\Delta \Psi \mathrm{m}(\mathrm{h} \Delta \Psi \mathrm{m}: 1 \Delta \Psi \mathrm{m})$ ratio of fresh and freeze-thaw semen between 40 minutes $(0)$ and 80 minutes (1) incubation within bulls. Bars with the superscripts are significantly different between incubation times; $\mathrm{a}=\mathrm{P}<0.05 ; \mathrm{a}=\mathrm{P}<0.01$.

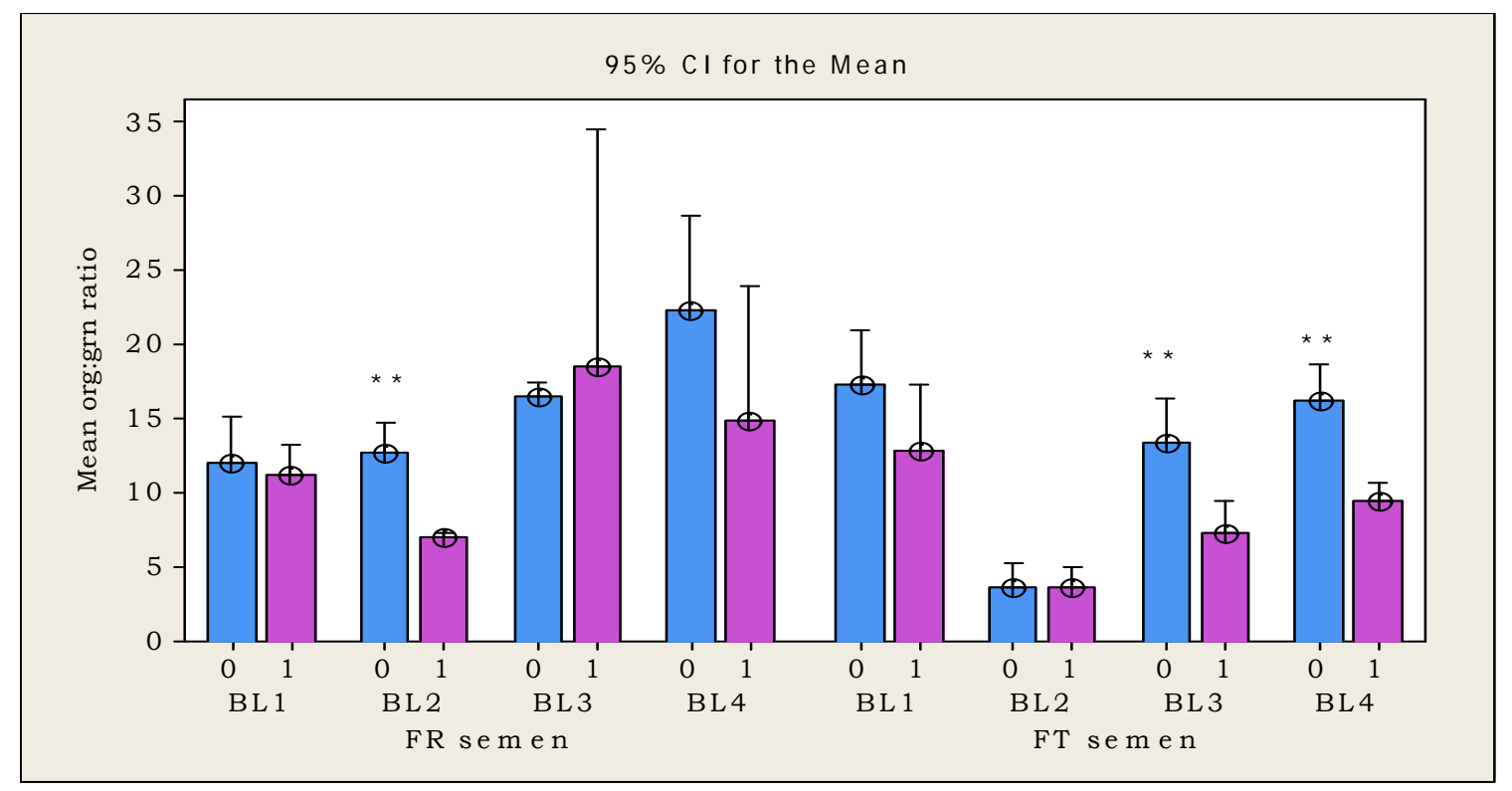

Fig. (6). Comparison of high: low $\Delta \Psi \mathrm{m}(\mathrm{h} \Delta \Psi \mathrm{m}: 1 \Delta \Psi \mathrm{m})$ ratio of fresh and freeze-thaw semen between 40 minutes $(0)$ and 80 minutes (1) incubation within bulls. Bars with the superscripts are significantly different between incubation times; ${ }^{* *}=\mathrm{P}<0.01$.

be the IAM effect on mitochondria or freeze/thaw process that changed mitochondrial status, which lessen the JC-1 accumulation in sperm mitochondria, increase green fluorescence and ultimately reduced $\mathrm{h} \Delta \Psi \mathrm{m}: 1 \Delta \Psi \mathrm{m}$ ratio.

\section{Comparison of High: Low $\Delta \Psi \mathrm{m}$ Ratio Between Bulls}

In spermatozoa of fresh semen at 40 min incubation: a higher $\mathrm{h} \Delta \Psi \mathrm{m}: 1 \Delta \Psi \mathrm{m}$ ratio was observed in bull 4 for all treatments except DOG, while a lower ratio was seen in bull 1 and bull 2, at all treatment (Fig. 7a \& b). This ratio was only significant between bull one and four, bull four and all bulls and bull one, three and four for CON, IAM and VAL respectively (Fig. 7a \& b). Inconsistency in $\mathrm{h} \Delta \Psi \mathrm{m}: 1 \Delta \Psi \mathrm{m}$ ratio was observed between the bulls in freeze-thaw semen at each treatment and incubation times. At 40 min incubation $\mathrm{h} \Delta \Psi \mathrm{m}: 1 \Delta \Psi \mathrm{m}$ ratio of freeze-thaw spermatozoa was higher in bull 1 treated with CON and IAM, this was significant with bull 2 at CON level; and lower in bull 2 at CON, DOG and VAL treatments, which was only significant with bull 3 at DOG level (Fig. 7a \& b). It seemed that inconsistency in $\mathrm{h} \Delta \Psi \mathrm{m}: 1 \Delta \Psi \mathrm{m}$ ratio between the bulls is a characteristic of individual bulls rather than the effect of treatment or incubation time or sperm may switch energy demand from respiration to glycolysis for a while. 

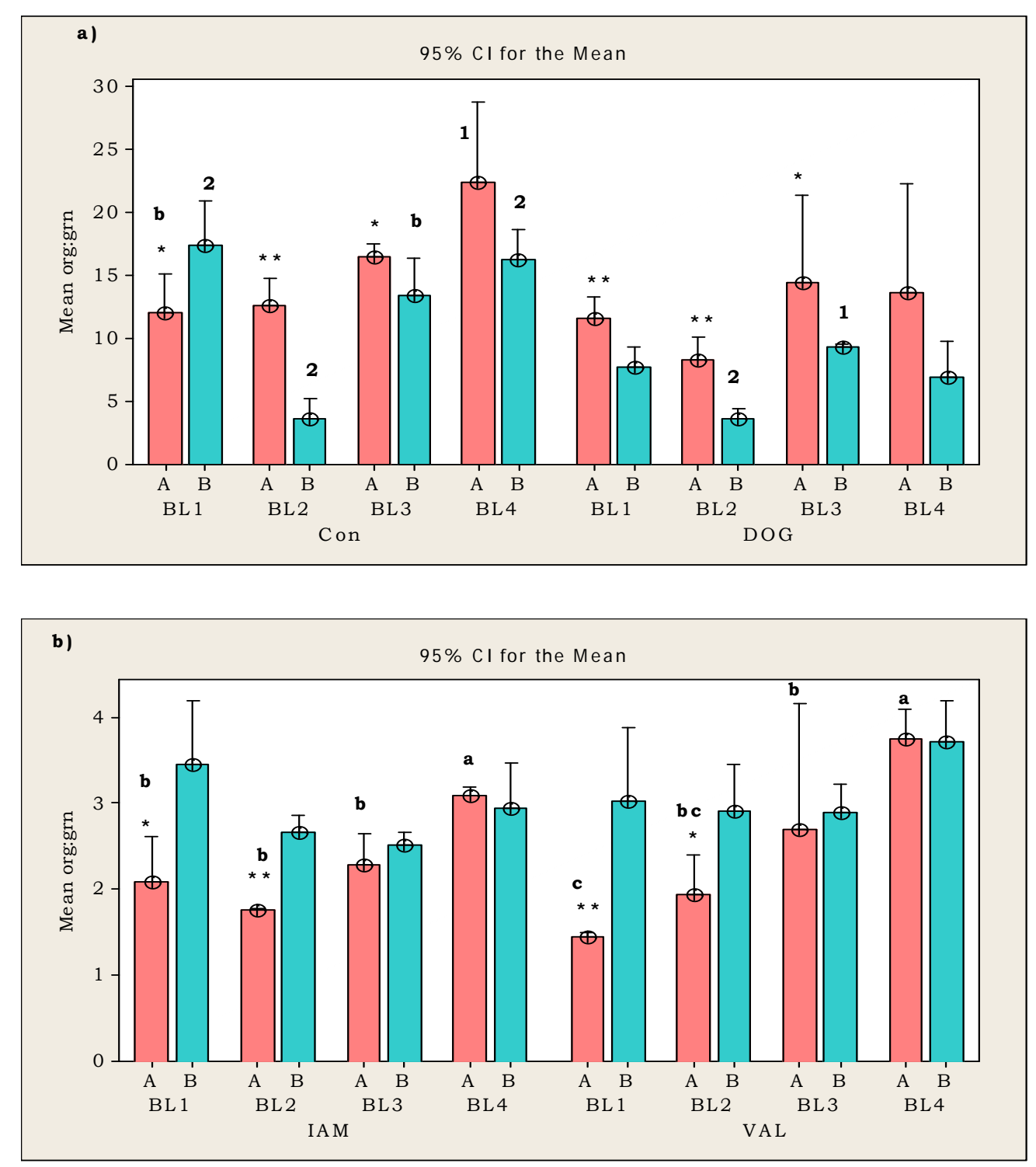

Fig. (7). (a \& b) Comparison of high: low $\Delta \Psi \mathrm{m}(\mathrm{h} \Delta \Psi \mathrm{m}: 1 \Delta \Psi \mathrm{m})$ ratio between bulls and fresh (A) and freeze-thaw (B) sperm within bulls at 40 minutes incubation. Bars with the superscripts are significantly different to freeze-thaw semen; $*=\mathrm{P}<0.05$ and $* *=\mathrm{P}<0.01$. $\mathrm{B}$ ars that do not share a superscript letter are significantly different between bulls.

\section{DISCUSSION}

In this investigation, we demonstrated for the first time bovine sperm mitochondrial function following inhibition of glycolytic pathways in the presence of respiratory substrate in fresh and frozen-thawed semen. Each dot plot of treatments showed a single cell population of membrane intact spermatozoa and each population displayed both green and orange fluorescence but to varying degrees. The orange and green populations in dot plots were difficult to separate. Therefore examination of orange:green (high:low $\Delta \Psi \mathrm{m}$ ) fluorescence ratio was performed to integrate green and orange fluorescence value in fresh and post-thaw semen.

Bovine spermatozoa incubated with JC-1 exhibited significant differences between treatments at low and high $\Delta \Psi \mathrm{m}$ and incubation times. An increase in the population with active mitochondria has been observed in samples either provided with only glycolytic substrate (sorbitol) or with glycolytic inhibitor (DOG) and pyruvate. Similar results have been observed by Mukai and Okuno [12] when mouse sperm were incubated with $\mathrm{JC}-1$ in presence of either glucose or DOG and pyruvate. Consistency in $\Delta \Psi \mathrm{m}$ between treatments Control and DOG might be due to presence of sorbitol in the former treatment, which is reduced to fructose by the polyol pathway and fructose further reduced into pyruvate/lactate, providing respiratory substrate to sperm like the DOG treated sample. In addition the increase in $\Delta \Psi \mathrm{m}$ in DOG treated sperm may be due to inhibition of glycolysis which in turn may cause the sperm to force mitochondria to work at a higher level to generate energy and the reduction in $\Delta \Psi \mathrm{m}$ at $80 \mathrm{~min}$ incubation might be due to sperm having utilized all the respiratory substrate pyruvate as a marked reduction in seminal pyruvic acid has been observed after $60 \mathrm{~min}$ [25].

Addition of carbonyl cyanide m-chlorophenylhydrazone (CCCP) reduced the mitochondrial inner-membrane 
potential as an uncoupler demonstrated as green emission from mitochondria [12]. Change in state of JC-1 from aggregates to monomers has been observed in human sperm [26] and stallion sperm [27], using VAL to validate the JC-1 probe. In the present study, VAL reduced $\Delta \Psi \mathrm{m}$ in bull spermatozoa resulting in a shift in fluorescence from orange (high $\Delta \Psi \mathrm{m}$ ) to green (low $\Delta \Psi \mathrm{m}$ ). When subtracting the aggregate of valinomycin from control, the true value for mitochondrial potential was lower as compared to control (data not shown) indicating that valinomycin has not completely abolished mitochondrial activity and or the presence of aggregates fluorescence could be background fluorescence of JC-1 due to the sensitivity of the fluorescence detectors; it is unlikely that sperm populations would ever show zero fluorescence. Increase in VAL concentration may help to differentiate the mitochondrial and fluorochrome activity.

Hathaway [28] observed that IAM completely reduced respiration in sea urchin spermatozoa within $45 \mathrm{~min}$. IAM, an inhibitor of GAPDH, did not reduce motility in presence of pyruvate and lactate but inhibited ATP produced from glycolysis with no effect on ATP produced by mitochondrial respiration in mouse sperm [15]. Recently Schmidt and Dringen [17] found that IAM markedly reduced glutathione (GSH), cellular GAPDH and lowered cellular lactate production in astrocytes and reduction in GSH level resulted in loss of mitochondrial membrane potential in mice sperm [29]. It has been reported that the sperm mitochondrial cysteine-rich protein (SMCP) and phospholipid hydroperoxide glutathione peroxidise are the proteins localized in the mitochondrial capsule and enhances sperm motility [30]. Like GAPDH, IAM may alter SMCP, GSH level and decrease $\triangle \Psi \mathrm{m}$. SMCP gene knockout in mice sperm reduced sperm motility and fertility [31]. In our study, IAM disrupted the mitochondrial activity in the presence of pyruvate and also shifted fluorescence from orange (high $\Delta \Psi \mathrm{m})$ to green (low $\Delta \Psi \mathrm{m}$ ). It reveals that IAM might be involved in the reduction of mitochondrial activity or restricting pyruvate oxidation, therefore depriving mitochondria of respiratory substrate.

Freeze-thaw cycle is the basis of major changes in spermatozoa such as increases in osmotic and oxidative stress, and membrane permeability, thereby significant decrease in sperm viability and mitochondrial membrane potential [32, 33]. Using $\mathrm{JC}-1$ probe to evaluate the functional potential of mitochondria of bovine sperm, Celeghini et al., [34], observed that for the percentage of spermatozoa with greater mitochondrial function, potential was approximately five fold less in frozen-thawed semen than in fresh semen. Similar results were found by Arruda and his colleagues [35] in cattle semen after cryopreservation with Tris-egg-yolk extender and another extender containing glycerol. Disappearance and significant reduction in aggregates, shift to monomers and marked change in aggregates:monomers ratio was also observed in frozenthawed bull sperm $[33,36]$ suggesting that the bulls had low cryopreservation tolerance. In this study washed frozenthawed spermatozoa diluted in extender supporting glycolysis showed significant increase (30\%) in $\mathrm{h} \Delta \Psi \mathrm{m}: 1 \Delta \Psi \mathrm{m}$ ratio than that of the fresh spermatozoa in bull 1 but decrease (20 to $70 \%$ ) in remaining three bulls. Changes in sperm membrane occur during the freezing process, similar to those that occur during capacitation and may induce damage to the mitochondria $[22,37]$. Oxidative stress is another factor that influences sperm mitochondrial function during freeze-thaw process [9]. Motility, mitochondrial function, and viability are the most likely all interrelated aspects of the overall physiological status of the spermatozoa [38, 39]. Therefore high level of $\mathrm{h} \Delta \Psi \mathrm{m}: 1 \Delta \Psi \mathrm{m}$ in bull 1 might be due to the capacitation- like effect of freeze-thaw, which moved sperm towards hyperactivation and consequently a rise in mitochondrial activity, whereas low level in other three bulls perhaps be the sperm mitochondria encountered to oxidative stress produced on decrease in antioxidant level during a freeze/thaw cycle.

On comparing incubation times using $\mathrm{h} \Delta \Psi \mathrm{m}: 1 \Delta \Psi \mathrm{m}$ ratio as factor of variation: maximum $\mathrm{h} \Delta \Psi \mathrm{m}: 1 \Delta \Psi \mathrm{m}$ ratio observed at $40 \mathrm{~min}$ incubation in all treatments and slightly lowered with VAL. Within the bulls at control level, decrease in $\mathrm{h} \Delta \Psi \mathrm{m}: 1 \Delta \Psi \mathrm{m}$ ratio from 40 to $80 \mathrm{~min}$ has been observed in all bulls of fresh and freeze-thawed sperm but increased in bull 3 in fresh semen, suggesting that variation in $\mathrm{h} \Delta \Psi \mathrm{m}: 1 \Delta \Psi \mathrm{m}$ is a characteristic of individual bulls or sperm of bull 3 may initially fulfil their energy demand via glycolysis for a while. Similarly among the bulls the ratio of $\mathrm{h} \Delta \Psi \mathrm{m}: 1 \Delta \Psi \mathrm{m}$, was higher in bull 4 followed by bull 3 and comparable in bull 1 and 2 of fresh semen; and for frozenthawed semen, this ratio was comparable in bull 1, 2 and 4 but markedly reduced in bull 2 . Generally the commercial testing bull semen is based on post-thawed progressive motility, which has never correlated well with fertility [40, 41]; therefore highly functional mitochondria after prolonged incubation of any bull pre or post freeze/thaw cycle may be a good indicator of bull selection and fertility prediction.

\section{CONCLUSION}

Mitochondrial function was significantly decreased on freeze-thaw process. Maximum mitochondrial potential has been found at 40 min incubation in fresh and frozen-thawed semen, suggesting that the $40 \mathrm{~min}$ incubation will be satisfactory for assessment of mitochondrial membrane potential in future. Mitochondrial activity was markedly reduced in samples treated with IAM and VAL indicating that IAM which is a known glycolytic inhibitor has a similar effect to VAL, recognized as a respiratory inhibitor, which can be validated by looking at either sperm oxygen consumption or ATP production or motility in the presence of IAM.

\section{CONFLICT OF INTEREST}

The authors confirm that this article content has no conflicts of interest.

\section{ACKNOWLEDGEMENTS}

The author is grateful to the Livestock and Fisheries Department, Government of Sindh, Pakistan for financial support. The author is also thankful to Mr. Abdul Qadir Junejo, Director, Animal Breeding Sindh, for their constructive advice. 


\section{REFERENCES}

[1] Hammerstedt RH, Graham JK, Nolan JP. Cryopreservation of mammalian sperm: what we ask them to survive. J Androl 1990; 11: 73-88.

[2] Parks JE, Graham JK. Effects of cryopreservation procedures on sperm membranes. Theriogenology 1992; 38: 209-22.

[3] Holt WV. Alternative strategies for the long-term preservation of spermatozoa. Reprod Fertil Dev 1997; 9: 309-19.

[4] Mazur P. Freezing of living cells: mechanisms and implications. Am J Physiol 1984; 247: C125-C42.

[5] Drobnis EZ, Crowe LM, Berger T, Anchordoguy TJ, Overstreet JW, Crowe JH. Cold shock damage is due to lipid phase-transitions in cell-membranes - a demonstration using sperm as a model. J Exp Zool 1993; 265: 432-7.

[6] Holt WV. Fundamental aspects of sperm cryobiology: the importance of species and individual differences. Theriogenology 2000; 53: 47-58.

[7] Holt WV, North RD. Partially irreversible cold-induced lipid phase transitions in mammalian sperm plasma membrane domains: freeze-fracture study. J Exp Zool 1984; 230: 473-83.

[8] Guthrie HD, Welch GR, Long JA. Mitochondrial function and reactive oxygen species action in relation to boar motility. Theriogenology 2008; 70: 1209-15.

[9] Bilodeau JF, Chaterjee S, Sirard MA, Gagnon C. Levels of antioxidant defenses are decreased in bovine spermatozoa after a cycle of freezing and thawing. Mol Reprod Dev 2000; 55: 282-8.

[10] Lindemann CB, Fisher M, Lipton M. A comparative study of the effects of freezing and frozen storage on intact and demembranated bull spermatozoa. Cryobiology 1982; 19: 20-8.

[11] Fraczek M, Piasecka M, Gaczarzewicz D, et al. Membrane stability and mitochondrial activity of human ejaculated spermatozoa during in vitro experimental infection with Escherichia coli, Staphylococcus haemoly and Bacteroides ureolyticus. Andrologia 2012; 20: 1-15.

[12] Mukai C, Okuno M. Glycolysis plays a major role for adenosine triphosphate supplementation in mouse sperm flagellar movement. Biol Reprod 2004; 71: 540-7.

[13] Amaral A, Ramalho-Santos. J. Assessment of mitochondrial potential: implications for the correct monitoring of human sperm function. Int J Androl 2010; 33: e180-e6.

[14] Hiipakka RA, Hammerstedt RH. 2-Deoxyglucose transport and phosphorylation by bovine sperm. Biol Reprod 1978; 19: 368-79.

[15] Pasupuleti V. Role of glycolysis and respiration in sperm metabolism and motility. MS thesis. Kent State University, Kent, Ohio, 2007.

[16] Krzyzosiak J, Molan P, Vishwanath R. Measurements of bovine sperm velocities under true anaerobic and aerobic conditions. Anim Reprod Sci 1999; 55: 163-73.

[17] Schmidt MM, Dringen R. Differential effects of iodoacetamide and iodoacetate on glycolysis and glutathione metabolism of cultured astrocytes. Front Neuro 2009; 1: 1-10.

[18] Harris EJ, Cockrell R, Pressman BC. Induced and spontaneous movements of potassium ions into mitochondria. J Biochem 1966; 99: 200-13.

[19] Saris NEL, Andersson MA, Mikkola R, et al. Microbial toxin's effect on mitochondrial survival by increasing $\mathrm{K}^{+}$uptake. Toxicol Ind Health 2009; 25: 441-6.

[20] Andersson MA, Mikkola R, Kroppenstedt RM, et al. The mitochondrial toxin produced by Streptomyces griseaus strains isolated from an indoor environment is valinomycin. Appl Environ Microbiol 1998; 64: 4767-73.

[21] Garrett JAL, Revell SG, Leese HJ. ATP production by bovine spermatozoa and its relationship to semen fertilising ability. J Androl 2008; 29: 449-58.
[22] Watson PF. Recent developments and concepts in the cryopreservation of spermatozoa and the assessment of their postthawing function. Reprod Fertil Dev 1995; 7: 871-91.

[23] Revell SG, Glossop CE. A long-life ambient temperature diluent for boar semen. Anim Prod 1989; 48: 579-84.

[24] Woolley DM, Crockett RF, Groom WDI, Revell SG. A study of synchronisation between the flagella of bull spermatozoa, with related observations. J Exp Biol 2009; 212: 2215-23.

[25] Marden WGR. Source of endogenous pyruvic acid in bovine seminal fluid and utilization. J Dairy Sci 1961; 44: 1688-97.

[26] Troiano L, Granata AR, Cossarizza A, et al. Mitochondrial membrane potential and DNA stainability in human sperm cells: a flow cytometry analysis with implications for male infertility. Exp Cell Res 1998; 241: 384-93.

[27] Love CC, Thompson JA, Brinsko SP, et al. Relationship between stallion sperm motility and viability as detected by two fluorescence staining techniques using flow cytometry. Theriogenology 2003; 60: 1127-38.

[28] Hathaway RR. Activation of respiration in sea urchin spermatozoa by egg water. Biol Bull 1963; 125: 486-98.

[29] Perl A, Yueming Q, Kazim RC, et al. Transaldolase is essential for maintenance of the mitochondrial transmembrane potential and fertility of spermatozoa. Proc Natl Acad Sci USA 2006; 103: 14813-8.

[30] Hawthorne SK, Goodarzi G, Bagarova J, et al. Comparative genomics of the sperm mitochondria-associated cysteine-rich protein gene. Genomics 2006; 87: 382-91.

[31] Nayernia K, Ibrahim MA, Elke BG, et al. Asthenozoospermia in mice with targeted deletion of the sperm mitochondrion-associated cysteine-rich protein (SMCP) gene. Mol Cell Biol 2002; 22: 304652.

[32] Ortega-Ferrusola C, Sotillo-Galán Y, Varela-Fernández E, et al. Detection of apoptosis-like changes during the cryopreservation process in equine sperm. J Androl 2008; 29: 213-21.

[33] Garner DL, Thomas AC, Gravance CG. The effect of glycerol on the viability, mitochondrial function and acrosomal integrity of bovine spermatozoa. Reprod Dom Anim 1999; 34: 399-404.

[34] Celeghini ECC, Arruda RP, Andrade AFC, Nascimento J, Raphael CF, Rodrigues PHM. Effects that bovine sperm cryopreservation using two different extenders has on sperm membranes and chromatin. Anim Reprod Sci 2008; 104: 119-31.

[35] Arruda RP, Gonzalez FRA, Celeghini ECC, Raphael CF. Effects of cryopreservation using different freezing techniques and cryoprotectants on plasmatic, acrosomal and mitochondrial membranes of bovine spermatozoa. Acta Sci Vet 2005; 33: S329.

[36] Thomas CA, Garner DL, Dejarnette JM, Marshall CE. Effect of cryopreservation of bovine sperm organelle function and viability as determined by flow cytometry. Biol Reprod 1998; 58: 786-93.

[37] Nagy S, Jansen J, Topper EK, Gadella BM. A triple stain flow cytometric method to assess plasma and acrosome membrane integrity of cryopreserved bovine sperm immediately after thawing in presence of egg-yolk particles. Biol Reprod 2003; 68: 1828-35.

[38] Anderson MJ, Chapman SJ, Videan EN, et al. Functional evidence for differences in sperm competition in humans and chimpanzees. Am J Phys Anthrop 2007; 134: 274-80.

[39] Garner DL, Thomas AC, Joerg HW, Dejarnette JM, Marshall CE. Fluorometric assessments of mitochondrial function and viability in cryopreserved bovine spermatozoa. Biol Reprod 1997; 57: 1401-6.

[40] Holt WV, Van Look KJW. Concepts in sperm heterogeneity, sperm selection and sperm competition as biological foundations for laboratory tests of semen quality. Reproduction 2004; 127: 527-35.

[41] Rodriguez-Martinez H. Laboratory semen assessment and prediction of fertility: still utopia? Reprod Domest Anim 2003; 38: $312-8$. 